\title{
A water soluble probe with near infra-red two-photon absorption and polarity-induced fluorescence for cerebral vascular imaging
}

\author{
Julien Massin, ${ }^{a}$ Azzam Charaf-Eddin, ${ }^{b}$ Florence Appaix, ${ }^{c}$ Yann Bretonnière, ${ }^{a}$ Denis Jacquemin, ${ }^{b, d^{*}}$ \\ Boudewijn van der Sanden, ${ }^{c *}$ Cyrille Monnereau ${ }^{a *}$ and Chantal Andraud ${ }^{a}$
}

\author{
${ }_{5}$ Received (in $\left.X X X, X X X\right)$ Xth $X X X X X X X X X 20 X X$, Accepted $X$ th $X X X X X X X X X 20 X X$ \\ DOI: $10.1039 / b 000000 x$
}

A water soluble Lemke chromophore's derivative shows unusual polarity dependence of its emission efficiency, leading to a strong red-NIR fluorescence in water when fitted with appropriate watersolubilizing polymer chains. In this contribution, the synthesis of the chromophore is described. The 10 dependence of its fluorescence on solvent polarity is investigated experimentally then rationalized on the basis of $a b$ initio calculations. Finally, we demonstrate that this chromophore is a valuable candidate for in vivo two-photon imaging of the cerebral vasculature, with two-photon absorption and emissions in the biological transparency window.

\section{Introduction}

15 In recent years, two-photon laser scanning microscopy (TPLSM), especially intravital, has emerged as an essential tool in biomedical research and has nowadays become a routine technique to track biological processes down to the cellular level in the living animal. ${ }^{1-4}$ In this context, fluorophores presenting 20 electronic transitions of the "intramolecular charge transfer" (ICT) type are considered as a particularly appealing class of molecules. The peculiar linear (environmental sensitivity, solvatochromism ${ }^{5}$ ) and non-linear (two-photon absorption, ${ }^{6-8}$ second harmonic generation ${ }^{9}$ ) spectroscopic properties associated 25 to ICT transitions have been taken advantage of for the development of functional materials, including $\mathrm{pH}^{10,11}$ or polarity probes $^{12,13}$ and biosensors ${ }^{14,15}$ with one or two-photon induced fluorescence. These fluorophores are inherently lipophilic, because of their extended conjugated carbon backbone. ${ }^{6}$ Thus, 30 major efforts have been put on the improvement of their solubility in biological media and their biocompatibility. In that context, it has been shown by Müllen and coll. that controlled growth of a star-shaped polymer shell around a central fluorophore was a particularly effective strategy. ${ }^{16-18}$ Moreover, 35 we have recently given a proof of principle that such structures were valuable candidates for the vectorization of two-photon fluorophores into cells. ${ }^{19}$ We showed that the resulting structures showed good-to-very-good conservation of their two-photon brightness in aqueous medium, with emission wavelengths up to ${ }_{40} 570 \mathrm{~nm}$. This result was however not fully optimal for in-vivo two-photon imaging applications, since it is desirable that the probe features maximal fluorescence in the biological window, ie at a wavelength between $700-1000 \mathrm{~nm}^{20-22}$

In this range of wavelengths, it is well documented that the 45 fluorescence efficiency of strongly solvatochromic ICT chromophores is generally decreased. This can be explained on the basis of the energy gap law, which postulates that nonradiative decay kinetic constants increase exponentially upon decreasing emission energy. ${ }^{23-25}$ Moreover this energy gap 50 mediated quantum yield (QY) decrease is often amplified in polar and/or protic solvents because of enhanced electrostatic interactions of the latter with the chromophore's strongly polarized ICT excited state. ${ }^{26}$ Finally, as mentioned above, ICT chromophores are generally inherently hydrophobic, which can 55 lead to formation of molecular aggregate in protic solvents such as alcohols or a fortiori water and can give rise to multiple additional non-radiative decay pathways. ${ }^{27}$

In a recent paper, several of us reported a series of red emitting Lemke's fluorophores (named after their initial discoverer ${ }^{28}$ ) 60 based on an dicyanoisophorone motif, as solid state luminescent materials. $^{29}$ Beside their solid-state fluorescence, another spectroscopic property raised our interest: whereas these red fluorophores were hardly emitting in low-polarity solvents, like toluene, we noticed that their fluorescence QY gradually 65 increased upon increasing solvent polarity. We felt that this particular feature could be taken advantage of in the context of bio-imaging applications which require probes that feature strong emission intensity in physiological fluids, preferentially in the biological transparency window. ${ }^{30}, 31$ We must underline that 70 although Lemke's type chromophores have been extensively investigated in the context of second order nonlinear optics ${ }^{32}$ (second harmonic generation ${ }^{33}$ and electro-optic modulation ${ }^{34-36}$ ), their luminescence properties have surprisingly been only sparingly studied and mostly in the solid state, these compounds 75 being considered essentially non emissive in solution. ${ }^{29,37,38}$ Thus, in the present paper, we report the full study of the first example of a water soluble Lemke's chromophore oligomerised derivative (Lem-PHEA) and of its precursor (Lem-In). We show for the first time that both compounds display unusually strong 
polarity induced enhancements of their luminescence QY along with significant red-shift of their emission, to reach, in the case of Lem-PHEA, 0.25 at $674 \mathrm{~nm}$ in water. Moreover, based on theoretical calculations we suggest a possible explanation 5 regarding the origin of this peculiar behaviour, namely a solvent mediated change in the potential energy surface of the first electronically excited state of the molecule. This makes the latter a good candidate for in vivo two-photon imaging of the cerebral vascular in the biological transparency window.

\section{${ }_{10}$ Results and discussion}

\section{Synthesis}

Oligomerized compound Lem-PHEA was synthesized following the general methodology introduced in a previous paper ${ }^{19}$ and depicted in Scheme 1. In a first step, a bromoisobutyryl moiety, 15 which is classically used as a polymerization initiator in ATRP synthesis, was introduced through a simple, high yielding esterification procedure on the two terminal hydroxyl groups of Lem-OH precursor (which was itself obtained by wellestablished literature procedures). The resulting compound, Lem-

${ }_{20}$ In, was then involved in an ATRP polymerisation procedure with (hydroxyethyl)acrylate (HEA) used as a monomer, a $\mathrm{Cu}$ (II)/bipy catalyst, and THF as a co-solvent for the reaction. This procedure allowed to obtain an oligomerized object with precise control over chain length ( $c a$ 10-12 monomer unit on each arm, as ${ }^{25}$ calculated from ${ }^{1} \mathrm{H}$ NMR integrations) as evidenced by ${ }^{1} \mathrm{H}$ NMR and GPC (Figures S8). Lem-PHEA and its precursors Lem-OH and Lem-In were further characterized by ${ }^{1} \mathrm{H}$ and ${ }^{13} \mathrm{C} \mathrm{NMR}$ (Figure S7), IR, UV-vis and fluorescence spectroscopy (see experimental for details).

30

\section{Linear spectroscopy: experiments}

Spectroscopic measurements were next undertaken on the polymeric Lem-PHEA and its 35 precursor Lem-In. As mentioned in introduction, solution luminescence spectroscopy of this class of chromophores had never been 40 the object of systematic, detailed investigations in previous literature. Thus, this study was mainly aiming at elucidating some of the relation 45 that exists between the solvent dependent emission energy of the fluorophore and other key spectroscopic parameters of the later (spectral bandwith, 50 fluorescence quantum yield, Stokes shift...), in order to better understand the origin of its polarity induced emission.

To this end, Lem-In and Lem${ }_{55}$ PHEA were studied in different

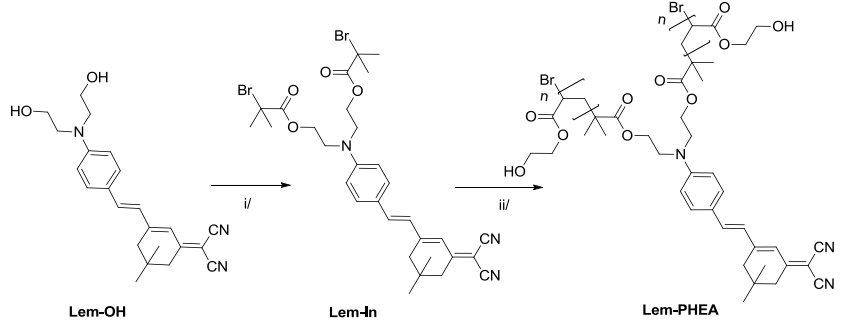

Scheme 1. Synthetic pathway; reagents and conditions: (i) 2bromoisobutyril bromide (3 eq.), Pyridine / THF 1:1, RT, $1 \mathrm{H}$ (ii) $\mathrm{CuBr}$ (1eq.), bipyridine (2 eq.), 2-hydroxyethylacrylate (HEA) /THF (1:1), $85^{\circ} \mathrm{C}, 20 \mathrm{~min}$

solvents that were chosen to cover the largest possible range of polarity, viscosities, and protic or non-protic character. Absorption and fluorescence emission spectra of both compounds were systematically recorded. Quantum yields (QY) were 60 calculated from integration of the fluorescence intensity at various concentrations and comparison to a standard (erythrosine). Absorption and emission curves are reported in Figure 1 whereas the main spectroscopic values are listed in Table 1.

65

First of all, for both compounds, broad, structureless emission bands were observed in all solvents of the study, which confirms the ICT character of the emission. The tendency observed qualitatively in our previous report (see Introduction) was fully 70 verified for both objects: upon increasing solvent's polarity (according to Lippert's polarity scale) from toluene to DMSO for Lem-In and from dioxane to water for Lem-PHEA, a significant red-shift of the emission energy was observed (positive solvatochromism), which was accompanied with a marked 75 increase of fluorescence QY (negative solvatokinetic) along with progressive narrowing of the emission band. A plot of the
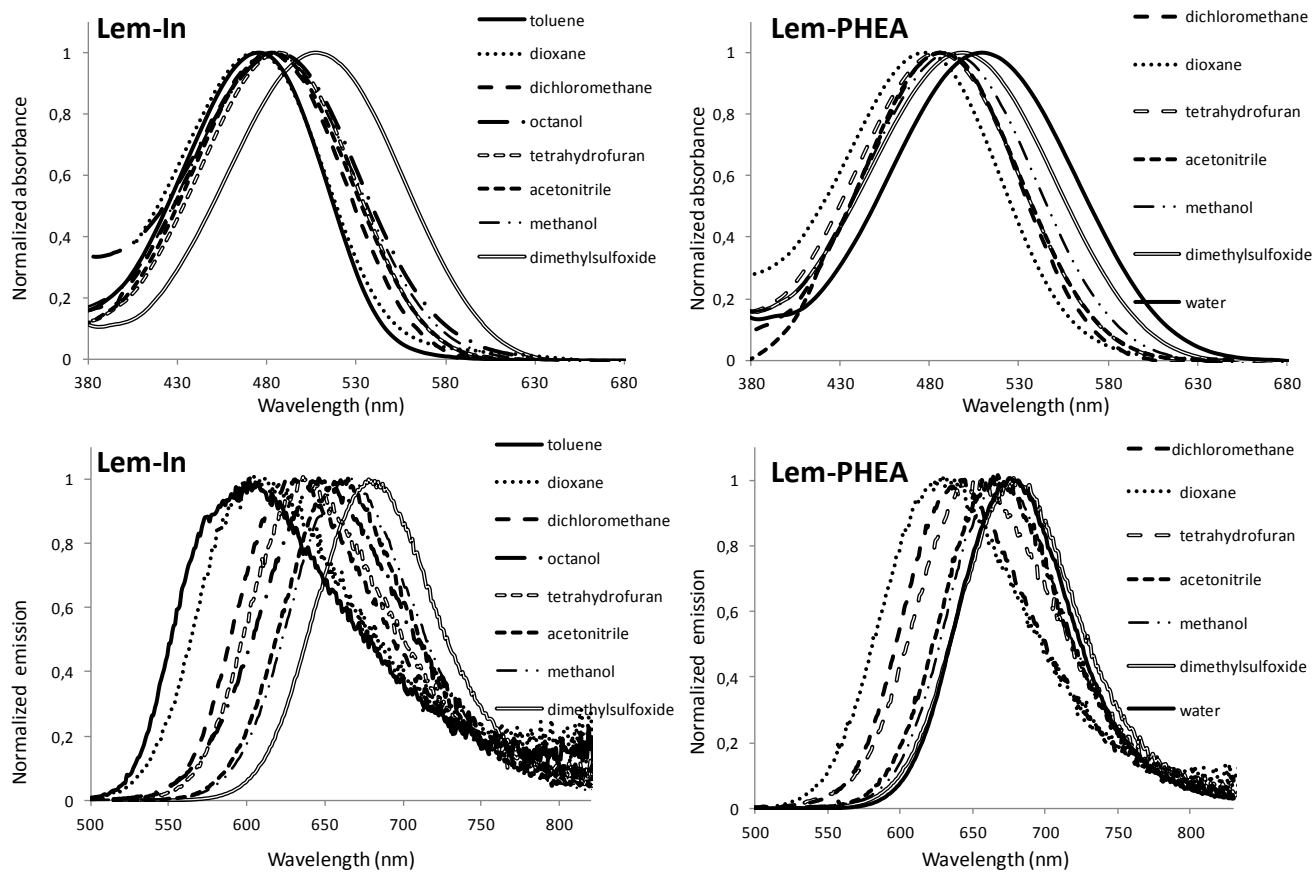

Figure 1. Normalized absorption (top) and emission (bottom) spectra of Lem-In (left column) and Lem-PHEA (right column) in the solvents of the study 


\begin{tabular}{|c|c|c|c|c|c|c|c|c|c|c|}
\hline & Solvent & $\Delta \mathrm{f}$ & $\lambda_{\max }(\mathrm{nm})$ & $\lambda_{\mathrm{em}}(\mathrm{nm})$ & $\Delta \bar{F}\left(\mathrm{~cm}^{-1}\right)$ & $\begin{array}{c}\Delta v_{1 / 2} \\
\left(\mathrm{~cm}^{-1}\right)\end{array}$ & $\phi_{\mathrm{f}}^{\mathrm{a}}$ & $\tau_{\mathrm{f}}(\mathrm{ns})^{\mathrm{b}}$ & $\begin{array}{c}\varepsilon \\
\left(\mathrm{M}^{-1} \cdot \mathrm{cm}^{-1}\right)\end{array}$ & $\begin{array}{c}\sigma_{\mathrm{TPA}} \\
(\mathrm{GM}) \\
\end{array}$ \\
\hline \multirow[t]{9}{*}{ Lem-in } & toluene & 0.013 & 474 & 602 & 4490 & 3300 & 0.008 & $<0.1$ & 27900 & - \\
\hline & dioxane & 0.02 & 474 & 606 & 4600 & 3080 & 0.016 & 0.13 & - & - \\
\hline & $\mathrm{CH}_{2} \mathrm{Cl}_{2}$ & 0.21 & 482 & 630 & 4870 & 2550 & 0.025 & 0.2 & 28200 & 530 \\
\hline & $\mathrm{CHCl}_{3}$ & 0.14 & 482 & 633 & 4950 & 2520 & 0.016 & 0.24 & - & - \\
\hline & TFH & 0.21 & 486 & 637 & 4877 & 2440 & 0.04 & 0.3 & - & - \\
\hline & octanol & 0.22 & 485 & 646 & 5140 & 2412 & 0.045 & 0.66 & - & - \\
\hline & $\mathrm{AcCN}$ & 0.305 & 484 & 661 & 5530 & 2050 & 0.07 & 0.5 & - & - \\
\hline & $\mathrm{MeOH}$ & 0.31 & 484 & 664 & 5600 & 2045 & 0.1 & 0.7 & - & - \\
\hline & DMSO & 0.26 & 508 & 680 & 4979 & 1940 & 0.3 & 1.3 & 30200 & - \\
\hline \multirow{8}{*}{ Lem-PHEA } & dioxane & 0.02 & 478 & 630 & 5050 & 2920 & 0.049 & 0.48 & - & - \\
\hline & $\mathrm{CH}_{2} \mathrm{Cl}_{2}$ & 0.21 & 490 & 640 & 4780 & 2400 & 0.045 & 0.58 & - & - \\
\hline & $\mathrm{CHCl}_{3}$ & 0.14 & 494 & 642 & 4670 & 2680 & 0.032 & 0.53 & - & - \\
\hline & TFH & 0.21 & 486 & 652 & 5240 & 2562 & 0.083 & 0.65 & - & - \\
\hline & $\mathrm{AcCN}$ & 0.305 & 483 & 668 & 5730 & 2080 & 0.12 & 0.67 & - & - \\
\hline & $\mathrm{MeOH}$ & 0.31 & 494 & 670 & 5317 & 1980 & 0.14 & 0.86 & - & - \\
\hline & DMSO & 0.26 & 500 & 678 & 5250 & 2050 & 0.26 & 1.34 & - & - \\
\hline & $\mathrm{H}_{2} \mathrm{O}$ & 0.32 & 510 & 676 & 4810 & 1930 & 0.22 & 1.14 & 21000 & 440 \\
\hline
\end{tabular}

$\mathrm{a} / \lambda \mathrm{ex}=488 \mathrm{~nm}$, reference is erythrosine in $\mathrm{MeOH}(\phi r e f=0.09)$, error margin $\pm 5 \% \mathrm{~b} / \lambda \mathrm{ex}=490 \mathrm{~nm}$, error margin $\pm 5 \%$

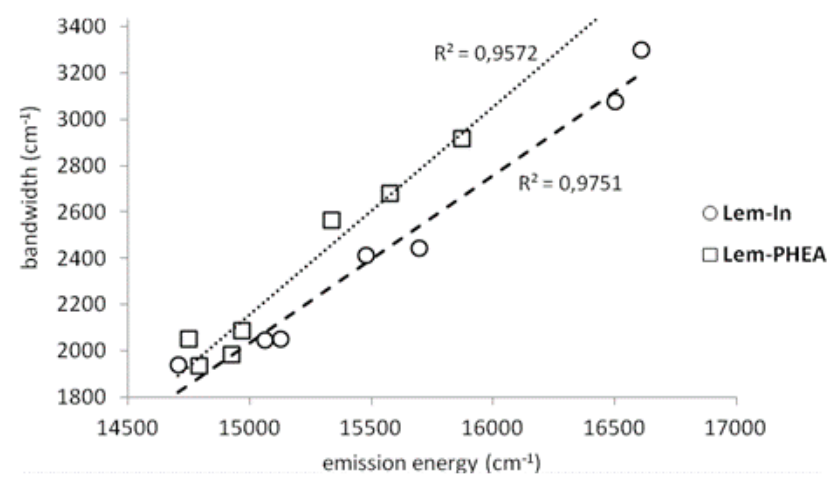

Figure 2. Plot of emission band-width vs energy at the maximum of the emission (as found in the solvatochromic study, Table 1). Linearization were performed independently for data obtained with Lem-In (dashed line) and Lem-PHEA (dotted line)

emission bandwidth versus emission energy clearly reveals, for Lem-In and Lem-PHEA, a close to linear dependence between those two parameters, with a bandwidth that narrows upon decreasing emission energy (Figure 2). Meanwhile, the band 5 shape becomes more symmetric as the emission energy decreases and a Gaussian shape is observed in most polar solvents (e.g. water and DMSO). Interestingly, this tendency is absolutely similar for Lem-PHEA and its precursor Lem-In. Such an evolution, which will be further commented in the following, is 10 opposed to what is classically observed for ICT fluorophores exhibiting positive solvatochromism: their emission band generally tends to become more broadened and structureless as solvent polarity increases, as a consequence of increased interactions with the solvent. $^{5}$

${ }_{15}$ Changes in emission energy and dependence of the Stokes shifts values on solvent polarity are important features in the context of ICT chromophores, since both parameters can be used in the engineering of polarity probes. ${ }^{5,}{ }^{39}$ On a more fundamental point of view, their study allows determining some important

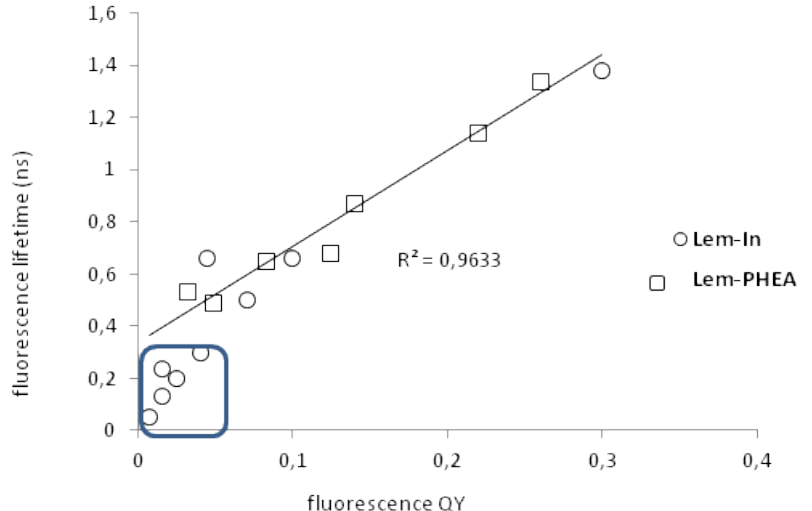

Figure 3. Plot of fluorescence quantum yield $v s$ excited state lifetime ((as found in the solvatochromic study, Table 1); data in the blue frame correspond to fluorescence lifetime $\leq 0.3 \mathrm{ns,} \mathrm{which} \mathrm{corresponds} \mathrm{to} \mathrm{the}$ lower limit of the detector, hence cannot be considered meaningful. Linear regression was performed on the joint data for Lem-In and

\section{Lem-PHEA}

${ }_{20}$ parameters of the molecule, like its dipolar moment at the ground $\left(\mu_{\mathrm{g}}\right)$ and excited $\left(\mu_{\mathrm{e}}\right)$ states and the electronic reorganisation (change in dipole moment $\mu_{\mathrm{CT}}$ ) that accompanies the transition between the ground and excited states. ${ }^{40,41}$ These parameters were thus investigated for Lem-In and Lem-PHEA. In the case 25 of Lem-In, absorption and emission energies as well as Stokes shift values showed a clear tendency to increase upon increasing solvent polarity (positive solvatochromism), thereby indicating an increased dipole moment at the excited state. This is consistent with what had been observed for related chromophores in our 30 previous contribution. ${ }^{29}$ The trend could be fitted with an acceptable correlation factor to Lippert-Mataga polarity scale ${ }^{13,42 \text {, }}$ ${ }^{43}$, (Figure S1) which allowed us to estimate dipole moments of the chromophore at its ground and excited states of $\mu_{\mathrm{g}}=10.5 \mathrm{D}$ and $\mu_{\mathrm{e}}=24.5 \mathrm{D}$, respectively, and a change in dipole moment $35 \mu_{\mathrm{CT}}=13.5 \mathrm{D}$. The situation is however not so straightforward to analyze for Lem-PHEA, since the polarity environment of the chromophore is in this case not only determined by the nature of 


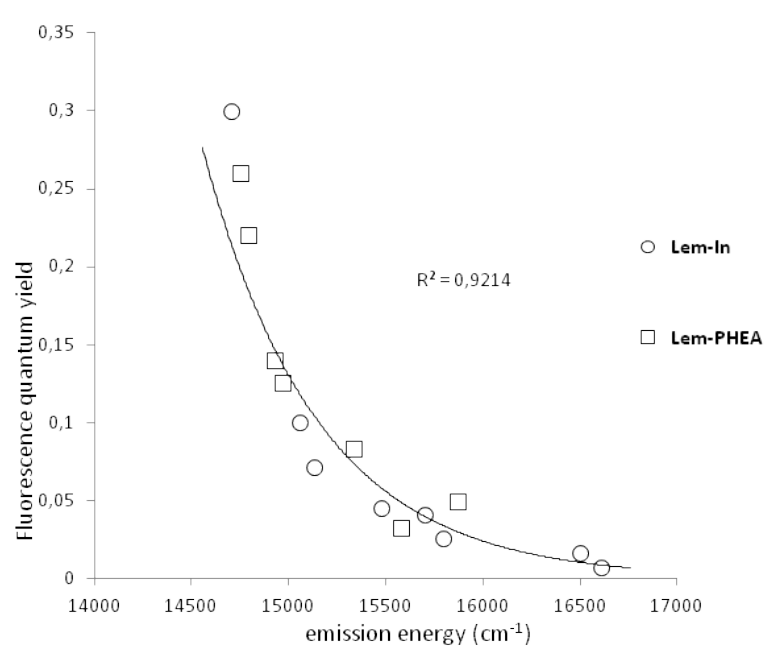

Figure 4. Plot of fluorescence quantum yield $v s$ emission energy (as found in the solvatochromic study, Table 1). Exponential fit was performed on the joint data for Lem-In and Lem-PHEA

the solvent, but by a more subtle interplay between chromophore/solvent and chromophore/polymer chain interactions. Indeed, it had been already established in our previous contribution devoted to alike water-soluble polymerized 5 chromophores ${ }^{19}$ that the polymer chain adopts different folding and orientations around the chromophore depending on the nature of the solvent. Thus, we were unable to identify a clear trend in Stokes shift and emission band position for Lem-PHEA (Figure S1).

10 Excited state lifetimes were also investigated for both systems in the same range of solvents as for QY measurements. A nearlinear dependence of fluorescence quantum yield on excited state lifetime could be noticed. The trend was so alike for both compounds that their data were fitted jointly (Figure 3). These 15 data allowed us to calculate radiative $\left(\mathrm{k}_{\mathrm{r}}\right)$ and non-radiative $\left(\mathrm{k}_{\mathrm{nr}}\right)$ kinetic constants for excited state decay (Figure S3): although $k_{r}$ slightly increases with solvent polarity, it remains in a comparable range in every solvent $\left(0.8-1.9 \times 10^{8} \mathrm{~s}^{-1}\right)$. In striking contrast, it can be noticed that a variation of more than one order 20 of magnitude is observed for $\mathrm{k}_{\mathrm{nr}}\left(1 \times 10^{10}\right.$ to $\left.7 \times 10^{8} \mathrm{~s}^{-1}\right)$. Altogether, this constitutes an unambiguous evidence that variations of the fluorescence quantum efficiency are mainly connected to modifications in the non-radiative excited state decay kinetic which decreases as polarity increases.

${ }_{25}$ An even more peculiar spectroscopic behaviour of these fluorophores was detected when plotting the evolution of their luminescence quantum efficiency (Figure 4) or alternatively effective. In Table S-1 in SI, we compare the XRD and DFT bond lengths fluorescence lifetime (see Figure S2) versus emission 30 energy. Whereas the energy gap law predicts an exponential increase of non-radiative excited state decay kinetics and therefore an exponential decrease of the quantum efficiency upon red-shift of the luminescence, ${ }^{24}$ the observed evolution for our dyes seems to follow a fully reverse trend. Indeed, the data 35 obtained for both Lem-In and Lem-PHEA could be jointly fitted with satisfactory accuracy $\left(r^{2}=0.921\right)$ to an inverse model, with an exponential increase of fluorescence lifetime and quantum efficiency upon luminescence red-shift. As mentioned before, this evolution is correlated with a significant sharpening of the 40 emission band. This is indicative that the vibronic contributions or the nature of the potential energy surfaces which result in the shoulder of the emission band in low-polarity solvents (see toluene), tend to vanish in polar ones (see DMSO). These changes also translate into the increased non-radiative 45 contribution to the ES decay observed in non-polar solvents (Figure S3). This point will be further discussed in the next section of this article. As a result, Lem-In and Lem-PHEA show a strongly negative solvatokinetic, ie their largest luminescence intensity was obtained in the most polar solvents of the study ${ }_{50}$ (DMSO for Lem-In, DMSO and water for Lem-PHEA). A closer examination of the data rules out simple explanations that could account for this peculiar behaviour, such as restriction of vibronic deactivations of the excited state through hydrogen bonding or solvent viscosity. On the one hand, hydrogen-bond 55 donor solvents, such as octanol, methanol and water perfectly follow the overall trend, and do not show any remarkable behaviour. On the other hand, solvents of close polarity, but of very different viscosities such as THF ( $c a \eta=0.5 \mathrm{cP}$ ) and octanol ( $c a \quad \eta=9 \mathrm{cP}$ ) show similar results in terms of fluorescence ${ }_{60}$ efficiency of the probes. An interesting consequence for biophotonic related applications is that, in contrast to most existing fluorescent bio-probes and polarity sensors, Lem-PHEA presents a luminescence that is turned on in a polar environment like blood or physiological fluid. This can be a very favourable 65 feature for angiographic applications as we will discuss latter.

\section{Linear spectroscopy : theoretical calculations}

To obtain further insights regarding the nature of the excitedstates, and to assess the origin of the experimentally observed polarity induced fluorescence Time-Dependent Density ${ }_{70}$ Functional Theory (TD-DFT) calculations have been performed on a model compound, referred to as Lem (Figure 5a, see Experimental Section for computational details). First, calculations were carried out with the least polar solvent, namely toluene, for which the selected continuum solvation model, which 75 does not account for any specific solute-solvent interaction(s), a/

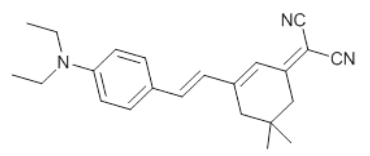

c/

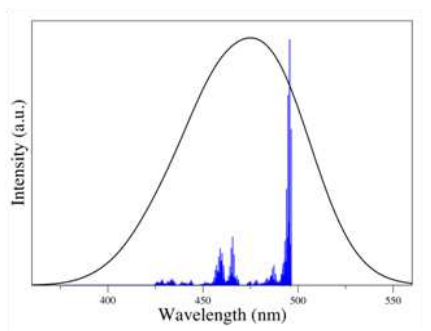

b/
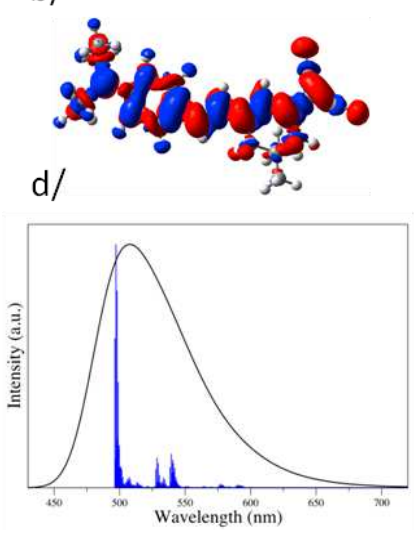

Figure 5: Left (a) Model compound (Lem) used for calculations (b) density difference plot computed in toluene: red (blue) zones correspond to increase (decrease) of density upon photon absorption (isovalue: $6 \times 10^{-}$

${ }^{4}$ a.u. (c) stick and convoluted vibrationally resolved absorption spectra in toluene.(d) stick and convoluted vibrationally resolved emission spectra in toluene. 


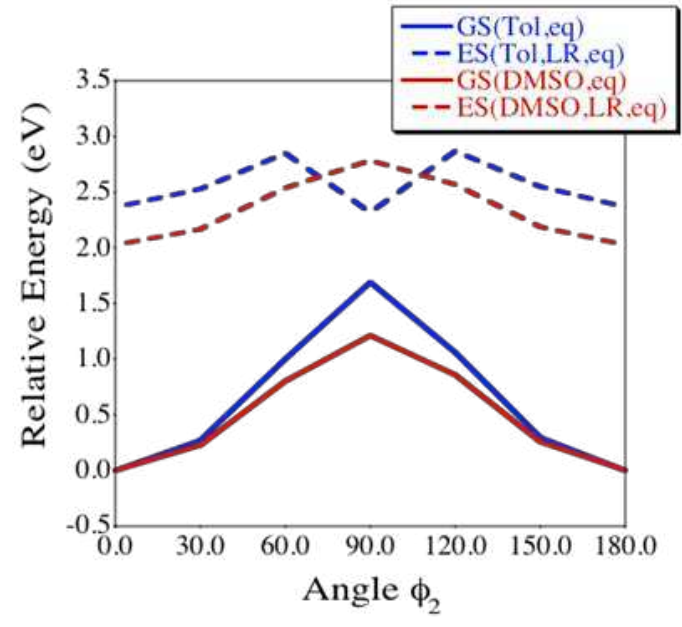

Figure 6: Evolution of the relative energies of the GS and ES of Lem in toluene and DMSO, for twist around $\phi_{2}$.

should be very measured along the $\pi$-conjugated path. As can be seen, there is a very good agreement between theory and experiment for both absolute distances (MAD $<0.01 \AA$ ) as well as the bond length alternation (BLA), which confirms the 5 accuracy of the selected level of theory. Moving from the ground to the lowest-lying excited state (which is the only state accessible in this range of energy, as confirmed by our calculations) induces a strong decrease of the BLA (-0.033 $⿱$ ) , which is characteristic of a more delocalized excited-state, as 10 expected. In the same vein, the density-difference plot (Figure 5 b) clearly confirms a strong CT character from the diethylamino side to the cyano groups, with a significant electronic reorganization on the connecting bridge. The ICT distance, estimated as the separation between the barycenters of electron 15 density gain/loss, reaches $3.75 \AA$ and is accompanied by a strong enlargement of the dipole moment $(+11.8 \mathrm{D})$ upon electronic transition, a trend that is in remarkable agreement with the experiment (see above). The vibrationally resolved absorption and emission spectra can be found in Figures $5 \mathrm{c}$ and $5 \mathrm{~d}$, 20 respectively. For absorption, one computes a maximum at 453 $\mathrm{nm}$, which is close to the experimental spot $(474 \mathrm{~nm})$. The theoretical FWHM is $3610 \mathrm{~cm}^{-1}$ for the absorption band. Apart from low-frequency vibrations close to the $0-0$ point, the width of the absorption band can be explained by a series of vibronic 25 couplings in the 430-445 $\mathrm{nm}$ domain. They imply to a complex mix of modes, but the strongest contributions correspond to intense stretching modes related to the alteration of the single/double bond nature along the conjugated skeleton. For instance, an effective conjugation coordinate (ECC) mode at $301607 \mathrm{~cm}^{-1}$ (in the ES) located on the central vinylic moiety is responsible for the most intense peak close to $430 \mathrm{~nm}$. Consistently with measurements, the fluorescence band is also asymmetric, and presents a FWHM of $2859 \mathrm{~cm}^{-1}$, which is slightly above the experimental reference $\left(3300 \mathrm{~cm}^{-1}\right.$, see Table

$351)$. However, the computed position of $\lambda_{\mathrm{em}}$ is $482 \mathrm{~nm}$, significantly off the experiment $(602 \mathrm{~nm})$ : the TD-DFT Stokes shift is clearly too small. In short, despite an undershot Stokes shift, theory reproduces nicely the band shapes in toluene. We have also simulated the same data in DMSO (Figure S6). In
${ }_{40} \mathrm{DMSO}$, the BLA is slightly smaller to the one computed in the apolar environment for the ground-state $(0.056 \AA$ in DMSO versus $0.061 \AA$ in toluene), the reverse holding for the excitedstate (0.034 $\AA$ in DMSO versus $0.028 \AA$ in toluene). Therefore the GS/ES variation of the main geometrical parameters related to 45 optical properties is smaller in DMSO than in toluene. However, the increase of dipole moment upon absorption (+13.5 D) significantly exceeds the toluene value. Moreover, we computed a FWHM of $3094 \mathrm{~cm}^{-1}$ for the fluorescence in DMSO, which is almost unchanged from toluene. This clearly departs from 50 experimental trends $\left(1800 \mathrm{~cm}^{-1}\right)$. Likewise, the fluorescence band is slightly asymmetric in the calculations (Figure S6), which also contrasts with the measurements. To estimate the importance of the non-radiative path, we determined the Huang-Rhys (HR) factors, following the procedure of Peng et al. ${ }^{44} \mathrm{We}$ found that 55 the HR factor decreases from toluene to DMSO. This indicates that non-radiative paths are less effective in the latter and therefore that fluorescence efficiency should increase. Although this result is in qualitative agreement with our experimental observations, the calculated effect $(-5 \%)$ appears significantly too 60 small to fully account for the dramatic experimental variation, which led us to envision other hypotheses. The theory/experiment differences might be ascribed to i) aggregation, ii) specific solutesolvent interactions iii) anharmonicity or iv) change in the nature of the potential energy surface that are not included in the ${ }_{65}$ previous model. The two first factors can be reasonably excluded: on the one hand, aggregation can be almost fully discarded on the basis of concentration-dependence studies on the absorption and fluorescence spectra; on the other hand, simple hydrogen-bonding can be ruled out on the basis of our experiments (vide supra). 70 Interestingly, the third factor (anharmonicity) is known to induce significant deviation from the standard energy-gap law, which could potentially account for the experimentally observed trend for the fluorescence QY. ${ }^{45}$ Rotation of the terminal groups at the ES could also account for the experimental trends (fourth factor). 75 Interestingly, investigations on this parameter revealed significant qualitative differences between toluene and DMSO (Figures S4S5, Figure 6). Indeed, while rotation around the dimethylamino moiety does not yield new minima on both GS and ES surfaces, the rotation around the dicyanovinylene group (angle $\phi_{2}$, Figure $\left.{ }_{80} \mathrm{~S} 4\right)$ yields a twisted minimum $\left(90^{\circ}\right)$ of the ES in toluene, that is absent from the ES in DMSO (Figure 6). Consequently, the ES of

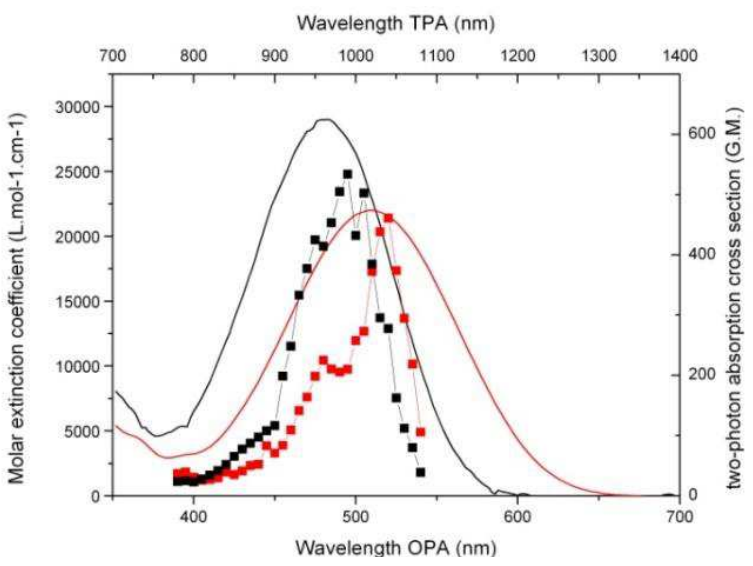

Figure 7. One-(full) and two-photon (full with marks) absorption spectra of Lem-In in dichloromethane (black) and Lem-PHEA in water (red) 
the emitter is predicted to be more rigid in DMSO than in toluene, which in turn could account for the difference of band shapes. As can be seen in Figure 6, this $90^{\circ}$ 5 structure presents an energetic minimum at the ES, slightly lower in energy than the planar conformation. Interestingly GS and ES energies of this $90^{\circ}$ structure are very close on the energetical scale, hinting a ${ }_{10}$ possible conical intersection ${ }^{46}$ that would offer a supplementary non-radiative pathway in toluene but not in DMSO. To say it differently, we suggest that the much lower ES-GS energy gap of the $90^{\circ}$ twisted 15 structure could explain its non-emissive nature. This leads us to conclude that the very strong reverse solvatokinetic effect observed experimentally cannot be fully explained by harmonic vibronic couplings,

20 or by electrostatic effects between the solute and the solvent, but is most likely related to a change of the surface potential at the ES.

\section{Two-photon absorption}

${ }_{25}$ Two-photon absorption properties of LemPHEA in water were evaluated and compared with those of its precursor LemIn in dichloromethane, in order to determine whether our strategy also 30 allowed an adequate conservation of this parameter. Two-photon absorption crosssections $\left(\sigma_{\mathrm{TPA}}\right)$ were measured using twophoton induced fluorescence experiments (see material and methods section for details). The results are reported in figure 7 ,

35 along with the linear absorption spectra Lem-In and Lem-PHEA in corresponding solvents. From these experiments, it is clear that only a slight decrease of $\sigma_{\mathrm{TPA}}$ occurs between Lem-In in dichloromethane and Lem-PHEA in water, which is found to reflect the evolution their respective linear molar extinction 40 coefficient. This demonstrates that Lem-PHEA, with a $\sigma_{\mathrm{TPA}}=$ $440 \mathrm{GM}$ and a maximal two photon brightness $\left(\sigma_{\mathrm{TPA}} \cdot \phi_{\mathrm{f}}\right)$ of about $100 \mathrm{GM}$, is unarguably a valuable candidate for the target application.

\section{In vivo two-photon microscopy}

${ }_{45}$ In order to evaluate its potential as bio-marker, Lem-PHEA was used in TPLSM of the functional cerebral vasculature in the motor cortex of mice brain, see materials and methods section. As it could be anticipated from the emission spectrum of the probe in water, its fluorescence emission intensity in vivo was higher in 50 the NIR region in comparison to the red region (Figure S9) for an excitation wavelength of $950 \mathrm{~nm}$. At this excitation wavelength no background endo-fluorescence of tissues was detected.

After intra-venous (i.v.) injection, Lem-PHEA circulated freely in the blood plasma with no adhesion to the vessel wall (Figure 558 a), This is important for, i) longitudinal imaging of the blood circulation, and ii) decreased long-term vascular toxicity. The red/NIR fluorescent emission in vivo is less diffused by brain tissues, which resulted in a maximal observation depth of $520 \mu \mathrm{m}$ in the adult mouse brain (Figure 8a) at normal laser power for in 60 vivo experiments: maximum $50 \mathrm{~mW}$ at the entrance of the objective.

In a time series experiment, the half-life time of Lem-PHEA in the blood plasma was estimated in the order of $11 \mathrm{~min}$ (see Figure $8 \mathrm{~b}-\mathrm{d})$. The clearance of the dye by the kidneys started already at ${ }_{65} 30 \mathrm{~min}$ after injection (Figure S10a). At this time the dye is observed in collecting tubules of the kidney and after two hours no dye was detected in the renal vascular compartment (Figure S10b-c). In the liver, Lem-PHEA stayed in the vascular compartment and was not found in hepatic cells for at least 2 70 hours after injection (Figure S10d). Thus renal clearance of LemPHEA is most probably preferred over liver metabolism of the dye. Such a fast renal clearance precludes its use for intravital microscopy studies of blood physiological phenomena exceeding 1 hour. On the other hand, it constitutes a very positive result 75 with regard to long-term toxicity of the compound in vivo. In the future, it could be envisioned to extend Lem-PHEA circulation time by enhancing polymer chains length, as already illustrated in the past for related systems. ${ }^{47}$

\section{Experimental}

\section{${ }_{80}$ Materials and methods}

Synthesis 
All solvents were used as received from the supplier. 2bromoisobutyryl bromide and bipyridine are commercially available and used as received from the suppliers. Copper (I) bromide (Aldrich, 98\%) was suspended in glacial acetic acid, 5 filtered and washed with acetic acid then thoroughly rinsed with ethanol . After extensive drying under vacuum it was stored at $4^{\circ} \mathrm{C}$ under argon. Hydroxyethylacrylate (HEA) was passed through a short pad of alumina prior to use. (E)-2,2'-(4-(2-(3Dicyanomethylene)-5,5-dimethylcyclohex-1-

10 enyl)(vinyl)phenylazanediyl)bis(ethane)bis(ethane-2,1-

diyl)diacetate was synthesized according to previouly reported literature methodologies. ${ }^{29}$ Column Chromatographies were performed on Merck Gerduran $60(40-63 \mu \mathrm{m})$. NMR spectra $\left({ }^{1} \mathrm{H}\right.$, ${ }^{13} \mathrm{C}$ ) of the monomers and polymers were recorded at room 15 temperature on a BRUKER 200 AVANCE and BRUKER 500 respectively. Data are listed in parts per million (ppm) and are reported relative to tetramethylsilane $\left({ }^{1} \mathrm{H},{ }^{13} \mathrm{C}\right)$, residual solvent peaks being used as internal standard. FTIR spectra (ATR) were performed on solid samples using a JASCO FT/IR 4100 20 spectrometer equipped with a JASCO ATR PRO450-S ATR module. HRMS measurements were performed by ESI-TOF: a Bruker Daltonics ${ }^{\circledR}$ Micro TOF-Q II was used with a resolution of 8000 , in positive mode with a capillary tension of $4500 \mathrm{~V}$, a source temperature of $180^{\circ} \mathrm{C}$, and a cone tension of $60 \mathrm{~V}$. The 25 internal reference used for calibration was sodium formate. Elemental analyses were performed on a Flash EA1112 CHNS/O Thermo Electron micro-analyzer. Polymers were analyzed by size exclusion chromatography (SEC) in DMF $\left(+\mathrm{LiBr}, 1 \mathrm{~g} \cdot \mathrm{L}^{-1}\right)$ at 60 ${ }^{\circ} \mathrm{C}$, at a flow rate of $0.8 \mathrm{~mL} \cdot \mathrm{min}^{-1}$ and at a concentration close to 6 $30 \mathrm{mg} \cdot \mathrm{mL}^{-1}$ after filtration through $0.2 \mu \mathrm{m}$ pore size membrane. The steric exclusion was carried out on two PSS GRAM $1000 \AA$ columns $\left(8 \times 300 \mathrm{~mm}\right.$; separation limits: 1 to $\left.1000 \mathrm{~kg} \cdot \mathrm{mol}^{-1}\right)$ and one PSS GRAM $30 \AA(8 \times 300 \mathrm{~mm}$; separation limits: 0.1 to 10 $\mathrm{kg} \cdot \mathrm{mol}^{-1}$ ) coupled with a differential refractive index (RI) 35 detector, a viscosimeter (Viscotek, Dual 250) and a light scattering (LS) detector (MiniDawn from Wyatt Technology, laser $\lambda=690 \mathrm{~nm}$ at $90^{\circ}, 45^{\circ}$ and $135^{\circ}$ ). The polydispersity indexes (PDI $=M_{\mathrm{w}} / M_{\mathrm{n}}$ ) of the samples were derived from the RI signal by a calibration curve based on PMMA standards (Polymer ${ }_{40}$ Standards Service). The number-average molar mass $\left(M_{\mathrm{n}}\right)$ were calculated from RI signals with the OmniSEC 4.6 software. Polymer size (Mn) was also calculated with ${ }^{1} \mathrm{H}$ spectroscopy, by comparison of the integration values found for the methyl endgroups of the initiator (signal "a" of the polymer, SI), and for the 45 side chain hydroxyethyl group (signals "d" and "e" of the polymer, SI).

\section{Spectroscopy}

UV-Visible spectra were recorded with a Jasco 670 UV-Visible spectrophotometer. The luminescence spectra were measured 50 using a Horiba-Jobin Yvon Fluorolog-3® spectrofluorimeter. The steady-state luminescence was excited by the unpolarised light from a $450 \mathrm{~W}$ xenon CW lamp and detected at an angle of $90^{\circ}$ for diluted solution measurements (10 mm quartz cuvette) by a redsensitive Hamamatsu R928 photomultiplier tube. Spectra were 55 reference corrected for both the excitation source light intensity variation (lamp and grating) and the emission spectral response (detector and grating). Fluorescence quantum yields $\mathrm{Q}$ were measured in diluted water solutions with an optical density lower than 0.1 using the relative method based on following equation: ${ }_{60} \mathrm{Qx} / \mathrm{Qr}=[\operatorname{Ar}(\lambda) / \mathrm{Ax}(\lambda)]\left[\mathrm{nx}^{2} / \mathrm{nr}^{2}\right][\mathrm{Dx} / \mathrm{Dr}]$

where $\mathrm{A}$ is the absorbance at the excitation wavelength $(\lambda), \mathrm{n}$ the refractive index and D the integrated luminescence intensity. " $r$ " and " $x$ " stand for reference and sample. Here, reference is erythrosine in $\mathrm{MeOH}(\mathrm{Qr}=0.45)$ at $488 \mathrm{~nm}$. Excitation of 65 reference and sample compounds was performed at the same wavelength. The TPA cross-section spectra were obtained by two-photon excited fluorescence measurement of dilute dichloromethane solutions of the compound using a Ti:sapphire femtosecond laser in the range 700-900 $\mathrm{nm}$. The excitation beam $70(5 \mathrm{~mm}$ diameter) was focalized with a lens (focal length $10 \mathrm{~cm})$ at the middle of the fluorescence cell $(10 \mathrm{~mm})$. The fluorescence, collected at $90^{\circ}$ to the excitation beam, was focused into an optical fiber (diameter $600 \mu \mathrm{m}$ ) connected to an Ocean Optics S2000 spectrometer. The incident beam intensity was adjusted to $7550 \mathrm{~mW}$ in order to ensure an intensity-squared dependence of the fluorescence over the whole spectral range. The detector integration time was fixed to $1 \mathrm{~s}$. Calibration of the spectra was performed by comparison with the published 700-900 nm Coumarin-307 and fluorescein two photon absorption spectra. ${ }^{48}$ 80 The measurements were done at room temperature in dichloromethane and at a concentration of $10^{-4}$ or $10^{-5} \mathrm{M}$.

\section{Calculations}

TD-DFT was used to probe the nature of the excited-states of the ${ }_{85}$ Lem dyes where the only structural simplification was the use of $\mathrm{NEt}_{2}$ donating group (see Figure 6). We have applied a computational protocol similar to the one applied in a recent benchmark paper ${ }^{49}$ and we redirect the interested reader to this contribution for further technical details and discussion. The 90 ground and excited-state structures have been optimized using analytic DFT and TD-DFT gradients, respectively. These force minimizations have been performed until the residual mean square force is smaller than $1 \times 10^{-5}$ a.u. The harmonic vibrational frequencies of both states have been computed on the optimal 95 geometries using analytic and numerical differentiation for GS and ES, respectively. This allowed to ascertain the nature of the minima but also to determine vibronic couplings. The vibrationally resolved spectra -within the harmonic approximation- were computed using the FCclasses program ${ }^{50,51}$ 100 The reported spectra have been simulated at $298 \mathrm{~K}$ using a convoluting Gaussian functions presenting a full width at halfmaximum (fwhm) of $0.06 \mathrm{eV}$. A maximal number of 25 overtones for each mode and 20 combination bands on each pair of modes were included in the calculation. The maximum number 105 of integrals to be computed for each class was set to $1 \times 10^{6}$. In our calculations, the electrostatic interactions between the dye and the environment have been modelled thanks to the PCM model that restores valid solvent effect, as long as no specific solute-solvent interactions take place. ${ }^{52}$ The adiabatic energies and consequently 110 the position of both absorption and fluorescence maxima in vibrationally resolved spectra have been evaluated using the state-specific (SS) PCM model that allows to correctly polarize the excited-state cavity ${ }^{53}$ Again, we redirect the interested reader to Ref. ${ }^{49}$ for a detailed discussion on the procedure used to 115 determine the adiabatic point. All our calculations have been performed with the Gaussian 09.A02 program $^{54}$ using the CAMB3LYP functional ${ }^{55}$ and the $6-31+\mathrm{G}(\mathrm{d})$ atomic basis set that are 
known to be a valuable combination for TD-DFT calculations. ${ }^{49}$ The ICT parameters have been computed using a recently proposed density-based model. ${ }^{56}$

\section{In-vivo two-photon microscopy}

In accordance with the policy of Grenoble Institute of Neuroscience (GIN) and the French legislation, experiments were done in compliance with the European Community Council Directive of November 24, 1986 (86/609/EEC). The research 10 involving animals was authorized by the Direction Départementale des Services Vétérinaires de l'Isère - Ministère de l'Agriculture et de la Pêche, France and the Direction Départementale de la protection des populations - Préfecture de l'Isère-France (F. Appaix, PhD, permit number 3809 39). All 15 efforts were made to minimize the number of mice used and their suffering during the experimental procedure. CD1 Mice were housed in cages with food and water ad libitum in a $12 \mathrm{~h}$ light/dark cycle at $22 \pm 1{ }^{\circ} \mathrm{C}$.

For in vivo TPLSM, 6 months old CD1 mice $(n=4)$ were 20 anesthetized using isoflurane (5\% for induction and 1-2\% during experiments) in a $70 \%$ air, $30 \% \mathrm{O}_{2}$ gas mixture. Their body temperature was monitored with a rectal probe and maintained at $36^{\circ} \mathrm{C}$ using a heating blanket. A catheter (Neoflon ${ }^{\mathrm{TM}}, \mathrm{BD}, \mathrm{USA}$ ) was inserted in the tail vein for an intravenous (iv) injection of $250.1 \mathrm{ml}$ Lem-PHEA solution at a concentration of $4 \mathrm{mg} / \mathrm{ml}$ just before the imaging experiments. For the two-photon imaging of the cerebral vasculature, a craniotomy of $2-3 \mathrm{~mm}$ in diameter was performed with a surgical drill above the motor cortex and filled with ultrasound gel. (The head was fixed in a homebuilt 30 stereotactic frame.)

Two-photon microscopy was performed using a LSM 7 MP (Zeiss, Germany) equipped with a 20x water-immersion objective (NA 1.0; Zeiss) and ZEN 2010 software. Laser excitation at 950 nm was done with a Ti:Sapphire laser system (Chameleon Ultra 35 II; Coherent, UK). Red Fluorescence light emission was collected in the epifluorescence configuration using a non-descanned photomultiplier tube with a 617/73 nm filter (Semrock, US). Most two-photon images were acquired as z-stacks with a maximum number of 257 images using the motorized objective 40 with a minimal step of $2 \mu \mathrm{m}$ between the different $\mathrm{x}-\mathrm{y}$ planes (512 x 512 pixels, $607.28 \mu^{2}$ ) resulting in a maximum observation depth of $520 \mu \mathrm{m}$ in the motor cortex of an adult mouse. The reconstruction of z-projections: sum of fluorescence intensities of all focal planes in a $\mathrm{z}$-stack, or generation of $3 \mathrm{D}$ 45 images were performed with ImageJ software ${ }^{57}$.

\section{Syntheses}

Lem-OH

(E)-2,2'-(4-(2-(3-Dicyanomethylene)-5,5-dimethylcyclohex-1enyl)-(vinyl)phenylazanediyl)-bis(ethane)bis(ethane-2,1-

${ }_{50}$ diyl)diacetate $(18 \mathrm{mmol})$ was dissolved in about $20 \mathrm{~mL}$ of methanol. Then, a solution of $\mathrm{K}_{2} \mathrm{CO}_{3}(0.5 \mathrm{~g})$ dissolved in $\mathrm{H}_{2} \mathrm{O}(1$ $\mathrm{mL}$ ) was added and the mixture was stirred at room temperature. After 1 hour, the suspension was diluted with water $(100 \mathrm{~mL})$. The aqueous layer was extracted three times with $\mathrm{CH}_{2} \mathrm{Cl}_{2}$ $55(3 \times 50 \mathrm{~mL})$ and the combined organic phases were washed with brine $(50 \mathrm{~mL})$, dried over $\mathrm{MgSO}_{4}$, concentrated under reduced pressure and purified by flash column chromatography (ethyl acetate/pentane : 1/9) to obtain a pure dark reddish solid. Yield: $5.91 \mathrm{~g}(87 \%)$.
${ }_{60}^{1} \mathrm{H}$ NMR (d $\mathrm{d}_{6}$-DMSO): $\delta(\mathrm{ppm}) 7.37(\mathrm{~d}, J=9 \mathrm{~Hz}, 2 \mathrm{H}), 6.97(\mathrm{~m}, 2 \mathrm{H})$, $6.53(\mathrm{~m}, 3 \mathrm{H}), 4.61(\mathrm{~b}, 2 \mathrm{H}), 3.33(\mathrm{~m}, 8 \mathrm{H}), 2.35(\mathrm{~m}, 4 \mathrm{H}), 0.81(\mathrm{~s}, 6 \mathrm{H})$. ${ }^{13} \mathrm{C}$ NMR $\left(\mathrm{d}_{6}\right.$-DMSO): $\delta(\mathrm{ppm}) 170.3,158.0,150.2,139.2,130.5$, 124.2, 123.5, 120.5, 115.1, 114.3, 112.1, 58.6, 53.6, 42.8, 38.9, 32.1, 28.0. Anal.Calcd for $\mathrm{C}_{23} \mathrm{H}_{27} \mathrm{~N}_{3} \mathrm{O}_{2}+\mathrm{H}_{2} \mathrm{O}$ : C, 69.85; H, 7.39; ${ }_{65} \mathrm{~N}, 10.62$. Found: C, 69.51; H, 6.83; N, 10.43. HRSM (ES): [M+ $\mathrm{Na}]^{+} 400.1988$ (calcd 400.1995)

\section{Lem-In}

Lem-OH (200mg, $0.53 \mathrm{mmol}$ ) was dissolved in $5 \mathrm{~mL}$ of THF and $70130 \mu \mathrm{L}$ of pyridine $(1.65 \mathrm{mmol})$. To the resulting reddish solution were added dropwise $270 \mu \mathrm{L}$ of bromoisobutyryl bromide (500mg, $2.2 \mathrm{mmol}$ ), and the solution was stirred at room temperature for 1 hour. Then dichloromethane $(10 \mathrm{~mL})$ was added, and the solution was extracted with $20 \mathrm{~mL}$ of $0,1 \mathrm{M} \mathrm{HCl}$.

75 The organic layed was dried, and solvents were removed under reduced pressure. The residue (single spot on TLC) was purified by column chromatography on silica gel $\left(\mathrm{CH}_{2} \mathrm{Cl}_{2}\right)$, and a reddish solid was obtained (190 mg, 56\%)

${ }^{1} \mathrm{H} \quad \mathrm{NMR}\left(\mathrm{CDCl}_{3}\right): \delta(\mathrm{ppm}) 7.42(\mathrm{~d}, J=7.5 \mathrm{~Hz}, 2 \mathrm{H}), 7.00(\mathrm{~d}$, $\left.{ }_{80} J=16 \mathrm{~Hz}, 1 \mathrm{H}\right), 6.78(\mathrm{~m}, 4 \mathrm{H}), 4.32(\mathrm{t}, J=6 \mathrm{~Hz}, 4 \mathrm{H}), 3.75(\mathrm{t}, J=6 \mathrm{~Hz}$, 4H), 2.52 (s, 2H), 2.42 (s, 2H), 1.87 (s, 12H), 1.04 (s, 6H) . 13C NMR $\left(\mathrm{CDCl}_{3}\right): \delta(\mathrm{ppm}) 171.3,169.2,155.0,148.5,137.5,129.6$, 125.1, 124.7, 121.8, 114.0, 113.2, 112.3, 62.9, 55.4, 49.1, 42.9, 39.2, 31.9, 30.7, 28.0. Anal.Calcd for $\mathrm{C}_{31} \mathrm{H}_{37} \mathrm{~N}_{3} \mathrm{O}_{4} \mathrm{Br}_{2}$ : C, 55.12; ${ }_{85} \mathrm{H}, 5.52$; N, 6.22. Found: C, 55.36; H, 5.77; N, 5.71. HRSM (ES): $[\mathrm{M}+\mathrm{H}]^{+} 674.1219($ calcd 674.1224$)$

\section{Lem-PHEA}

Lem-In (45 mg, $0.066 \mathrm{mmol}$ ), $\mathrm{CuBr}$ (26 mg, $0.135 \mathrm{mmol}$ ), and 90 2,2'-bipyridine (42 mg, $2.68 \mathrm{mmol}$ ) were dissolved in a mixture of THF $(1 \mathrm{~mL})$ and HEA $(1 \mathrm{~mL}, 7.7 \mathrm{mmol})$. Argon was bubbled for 15 minutes, the tube was sealed, and the resulting reddish solution was stirred for 17 minutes at $85^{\circ} \mathrm{C}$. Then, the solution was cooled rapidly by immersion in liquid nitrogen for 15 95 seconds, the tube was opened, its content was diluted in ca 10 $\mathrm{mL}$ of MilliQ water, put in a dialysis bag $(\mathrm{MWCO}=1000 \mathrm{Da})$ and dialized against a large volume of MilliQ water (which was replaced at regular intervals) for 2 days. The resulting reddish solution was lyophilized yielding $120 \mathrm{mg}$ of a fibrous solid.

${ }_{100}{ }^{1} \mathrm{H} \operatorname{NMR}\left(\mathrm{CD}_{3} \mathrm{OD}\right): \delta(\mathrm{ppm}) 7.5-6.75(\mathrm{~m}, 7 \mathrm{H}), 4.13(\mathrm{~b}, \approx 40 \mathrm{H})$, $3.73(\mathrm{~b}, \approx 40 \mathrm{H}), 2.38(\mathrm{~b}, \approx 20 \mathrm{H}), 1.75(\mathrm{~b}, \approx 40 \mathrm{H}) 1.10(\mathrm{~s}, 6 \mathrm{H}), 1.04$ $(\mathrm{s}, 12 \mathrm{H}) .{ }^{13} \mathrm{C} \mathrm{NMR}\left(\mathrm{CD}_{3} \mathrm{OD}\right): \delta(\mathrm{ppm}) 177.5,175.3,173.2$, $169.2,156.5,149.5,138.6,129.7,124.7,124.5,120.8,113.9$, 113.2, 111.3, 66.0, 62.0, 59.7, 53.5, 48.1, 42.6, 41.5, 38.7, 34.6, $10531.6,31.0,27.6,26.9$. GPC $\mathrm{Mn}=4737 \mathrm{Mw} / \mathrm{Mn}=2.08$

\section{Conclusions}

We reported the synthesis and the spectroscopic study of a Lemke chromophore derivative bearing two water-solubilizing polymeric arms. We showed that the latter was soluble in water and 110 physiological serum $(0.5 \%$ aq. $\mathrm{NaCl})$ above millimolar concentrations. The fluorescence is almost completely quenched, both for the polymeric object Lem-PHEA and its monomeric precursor Lem-In in low-polarity solvents, but strongly increases and is significantly red-shifted as solvents polarity is increased. 115 The origin of this very unusual behaviour, ie a positive solvatochromism along with a negative solvatokinetic effect, 
could be partly elucidated on the basis of theoretical calculations. It was shown by PCM-TD-DFT calculation that these effects were neither related to harmonic vibronic couplings nor to electrostatic interactions between the solute and the solvent, but 5 most probably to an excited-state twisting of the terminal dicyano group. Although further studies are probably necessary to fully quantify the origin of the deviation from the energy gap law that we clearly evidenced in this work, this behaviour made it possible to obtain an efficient water soluble two-photon probe with 10 absorption and polarity induced fluorescence in the NIR, with good quantum yield $\left(\phi_{\mathrm{f}}=0.25 @ 676 \mathrm{~nm}\right)$ and two-photon cross section $\left(\sigma_{\mathrm{TPA}}=440 \mathrm{GM} @ 1040 \mathrm{~nm}\right)$. Using TPLSM with this probe, we obtained three dimensional images of the functional cerebral vasculature in the motor cortex of mice, in a NIR-NIR 15 (excitation-emission) configuration, with very high contrast and resolution. Meanwhile, we also showed that the probe was cleared by the renal system with no accumulation in the hepatic cells. This constitutes a very promising feature in the context of in vivo imaging, especially in view of diagnostic applications. We 20 underline that, although Lemke chromophore derivatives had never been used previously, to the best of our knowledge, for biological imaging related applications a fortiori in vivo, the present contribution provides clear-cut evidences that this easily accessible class of molecules constitute valuable candidates for 25 two-photon bio-imaging applications in the NIR.

\section{Acknowledgments}

We thank Patrice L. Baldeck and J. Bernard for their precious help with two-photon induced fluorescence measurements (twophoton cross section determinations).We thank the Région 30 Rhône-Alpes (Cluster 5) for $\mathrm{PhD}$ grant (JM) and fundings. A.C.E. thanks the European Research Council (ERC, Marches 278845) for his post-doctoral grant. D.J. acknowledges the European Research Council (ERC) and the Région des Pays de la Loire for financial support in the framework of a Starting Grant (Marches 35278845 ) and a recrutement sur poste stratégique, respectively. This research used resources of 1) the GENCI-CINES/IDRIS (Grant c2012085117), 2) CCIPL (Centre de Calcul Intensif des Pays de Loire), 3) a local Troy cluster. The intravital microscopy studies were performed on the France Life Imaging Platform at ${ }_{40}$ Grenoble.

\section{Notes and references}

${ }^{a}$ Laboratoire de Chimie, UMR CNRS-5182,Université de Lyon, Université Claude Bernard, École Normale Supérieure de Lyon, 46 allée d'Italie, 69364 Lyon, France Tel: (+33)472728860

45 E-mail:xxxx@aaa.bbb.ccc

${ }^{b}$ CEISAM, UMR CNRS 6230, Université de Nantes, , 2 rue de la Houssinière, 44322 Nantes cedex 3, France. Fax: 33251125712; Tel:33251125564

${ }^{b}$ Grenoble Institute of Neuroscience(GIN), Inserm U 836 - Université 50 Joseph Fourrier - CEA - CHU, Chemin Fortuné Ferrini, 38042, La Tronche Cedex

${ }^{d}$ Institut Universitaire de France, 103, bd Saint-Michel, F-75005 Paris Cedex 05, France

$55 \dagger$ Electronic Supplementary Information (ESI) available: Polarity plot and calculation of the ground and excited state dipole moments. Plot of the emission lifetime and of radiative and nonradiative kinetic constants for the excited state decay vs emission energy. ${ }^{1} \mathrm{H}$ and ${ }^{13} \mathrm{C}$ NMR for LemOH, Lem-In and Lem-PHEA. Computed bond lengths for a model 60 molecule (Lem) at its ground and excited states . Dependence on the ground and excited state energy on choosen angular deformations within the molecule. GPC characterization of Lem-PHEA. Overlay of the emission of Lem-PHEA in water and the detector's ranges in Red and NIR configurations, and comparisons of the respective signal intensities 65 in red and NIR configuration. Pictures evidencing renal clearance of teh dye, and its absence of accumulation in hepatic cells See DOI: $10.1039 / \mathrm{b} 000000 \mathrm{x} /$

1. H. Kobayashi, M. Ogawa, R. Alford, P. L. Choyke and Y. Urano, Chem. Rev., 2009, 110, 2620-2640.

2. F. Leblond, S. C. Davis, P. A. Valdés and B. W. Pogue, $J$. Photochem. Photobiol. B, 2010, 98, 77-94.

3. J. Rao, A. Dragulescu-Andrasi and H. Yao, Curr. Opin. Biotechnol., 2007, 18, 17-25.

75 4. T. Terai and T. Nagano, Curr. Opin. Biotechnol., 2008, 12, 515-521.

5. C. Reichardt, Chem. Rev., 1994, 94, 2319-2358.

6. M. Albota, D. Beljonne, J.-L. Brédas, J. E. Ehrlich, J.-Y. Fu, A. A. Heikal, S. E. Hess, T. Kogej, M. D. Levin, S. R. Marder, D. McCordMaughon, J. W. Perry, H. Röckel, M. Rumi, G. Subramaniam, W. W. Webb, X.-L. Wu and C. Xu, Science, 1998, 281, 1653-1656.

7. J. L. Bredas, C. Adant, P. Tackx, A. Persoons and B. M. Pierce, Chem. Rev., 1994, 94, 243-278.

8. G. S. He, L.-S. Tan, Q. Zheng and P. N. Prasad, Chem. Rev., 2008 , 108, 1245-1330.

85 9. D. R. Kanis, M. A. Ratner and T. J. Marks, Chem. Rev., 1994, 94, 195-242.

10. D. Cui, X. Qian, F. Liu and R. Zhang, Org. Lett., 2004, 6, 2757-2760.

11. M.-H. Zheng, J.-Y. Jin, W. Sun and C.-H. Yan, New J. Chem., 2006, 30, 1192-1196.

90 12. M. Y. Berezin, H. Lee, W. Akers and S. Achilefu, Biophys. J., 2007, 93, 2892-2899.

13. R. W. Sinkeldam and Y. Tor, Org. Biomol. Chem., 2007, 5, $2523-$ 2528.

14. H. Cao and M. D. Heagy, J. Fluoresc., 2004, 14, 569-584.

95 15. Z. Xu, X. Qian and J. Cui, Org. Lett., 2005, 7, 3029-3032.

16. M. Yin, K. Ding, R. A. Gropeanu, J. Shen, R. d. Berger, T. Weil and K. Mullen, Biomacromolecules, 2008, 9, 3231-3238.

17. M. Yin, J. Shen, R. Gropeanu, G. O. Pflugfelder, T. Weil and K. Müllen, Small, 2008, 4, 894-898.

100 18. M. Yin, J. Shen, G. O. Pflugfelder and K. Mullen, J. Am. Chem Soc., 2008, 130, 7806-7807.

19. C. Monnereau, S. Marotte, P.-H. Lanoe, O. Maury, P. Baldeck, D. Kreher, A. Favier, M.-T. Charreyre, J. Marvel, Y. Leverrier and C. Andraud, New J. Chem., 2012, 36, 2328-2333.

105 20. W. Denk, J. Strickler and W. Webb, Science, 1990, 248, 73-76.

21. F. Helmchen and W. Denk, Nat. Methods, 2005, 2, 932-940.

22. S. A. Hilderbrand and R. Weissleder, Curr. Opin. Biotechnol., 2010 , 14, 71-79.

23. V. Chynwat and H. A. Frank, Chem. Phys., 1995, 194, 237-244

110 24. R. Englman and J. Jortner, Mol. Phys., 1970, 18, 145-164.

25. J. V. Caspar, E. M. Kober, B. P. Sullivan and T. J. Meyer, J. Am Chem Soc., 1982, 104, 630-632.

26. A. L. Macanita, F. P. Costa, S. M. B. Costa, E. C. Melo and H Santos, J. Phys. Chem., 1989, 93, 336-343.

115 27. M. H. Gehlen and F. C. De Schryver, Chem. Rev., 1993, 93, 199-221.

28. R. Lemke, Synthesis, 1974, 1974, 359-361.

29. J. Massin, W. Dayoub, J.-C. Mulatier, C. Aronica, Y. Bretonnière and C. Andraud, Chem. Mater., 2010, 23, 862-873.

30. D. R. Larson, W. R. Zipfel, R. M. Williams, S. W. Clark, M. P.

120 Bruchez, F. W. Wise and W. W. Webb, Science, 2003, 300, 1434 1436.

31. M. J. Levene, D. A. Dombeck, K. A. Kasischke, R. P. Molloy and W. W. Webb, J. Neurophys., 2004, 91, 1908-1912.

32. S. Ermer, S. M. Lovejoy, D. S. Leung, H. Warren, C. R. Moylan and

125 R. J. Twieg, Chem. Mater., 1997, 9, 1437-1442.

33. R. Andreu, S. Franco, E. Galán, J. Garín, N. Martínez de Baroja, C. Momblona, J. Orduna, R. Alicante and B. Villacampa, Tetrahedron Lett., 2010, 51, 3662-3665.

34. L. Chen, G. Qian, Y. Cui, X. Jin, Z. Wang and M. Wang, J. Phys. Chem. B, 2006, 110, 19176-19182.

35. I. Rau, P. Armatys, P. A. Chollet, F. Kajzar, Y. Bretonnière and C. Andraud, Chem. Phys. Lett., 2007, 442, 329-333. 
36. Q. Zhang, Y. Tu, H. Tian and H. Ågren, J. Phys. Chem. B, 2007, 111, 10645-10650.

37. J. Li, D. Liu, Z. Hong, S. Tong, P. Wang, C. Ma, O. Lengyel, C.-S. Lee, H.-L. Kwong and S. Lee, Chem. Mater., 2003, 15, 1486-1490.

5 38. L. Yang, M. Guan, D. Nie, B. Lou, Z. Liu, Z. Bian, J. Bian and C. Huang, Opt. Mater., 2007, 29, 1672-1679.

39. G. Weber and F. J. Farris, Biochemistry, 1979, 18, 3075-3078.

40. E. G. McRae, J. Phys. Chem., 1957, 61, 562-572.

41. E. Lippert, Ber. Bunsen Ges. Phys. Chem. , 1957, 61, 962-975.

10 42. P. Suppan, J. Photochem. Photobiol. A, 1990, 50, 293-330.

43. N. Mataga, Y. Kaifu and M. Koizumi, Bull. Chem. Soc. Jpn, 1956, 29, 465-470.

44. Q. Peng, Y. Yi, Z. Shuai and J. Shao, J. Am. Chem Soc., 2007, 129, 9333-9339.

15 45. R. F. Goldstein and W. Bialek, Phys. Rev. B, 1983, 27, 7431-7439.

46. although CI intersection between two states cannot be fully optimized with a LR-TD-DFT scheme the characteristic evolution of the GS and ES potential curves near $90^{\circ}$ twist angle constitutes good support to our hypothesis. Of couse, CAS or MR approaches that allow CI

20 optimization are both out of reach for a dye presenting a such a large conjugated backbone

47. M. Fox, E., F. Szoka and J. M. J. J. Fréchet, Acc. Chem. Res., 2009, 42, 1141-1151.

48. C. Xu and W. W. Webb, J. Opt. Soc. Am. B, 1996, 13, 481-491.

25 49. D. Jacquemin, A. Planchat, C. Adamo and B. Mennucci, J. Chem. Theory Comput., 2012, 8, 2359-2372.

50. F. Santoro, R. Improta, A. Lami, J. Bloino and V. Barone, J. Chem. Phys., 2007, 126, 084509-084513.

51. F. Santoro, A. Lami, R. Improta and V. Barone, J. Chem. Phys., 2007, 126, 184102-184111.

52. J. Tomasi, B. Mennucci and R. Cammi, Chem. Rev., 2005, 105, 2999-3094.

53. R. Improta, V. Barone, G. Scalmani and M. J. Frisch, J. Chem. Phys., 2006, 125, 054103-054109.

35 54. Frisch, M. J.; Trucks, G. W.; Schlegel, H. B.; Scuseria, G. E.;Robb, M. A.; Cheeseman, J. R.; Scalmani, G.; Barone, V.; Mennucci, B.; Petersson, G. A.; Nakatsuji, H.; Caricato, M.; Li, X.; Hratchian, H. P.; Izmaylov, A. F.; Bloino, J.; Zheng, G.; Sonnenberg, J. L.; Hada, M.; Ehara, M.; Toyota, K.; Fukuda, R.; Hasegawa, J.; Ishida, M.; Nakajima, T.; Honda, Y.; Kitao, O.; Nakai, H.; Vreven, T.; Montgomery, J. A., Jr.; Peralta, J. E.; Ogliaro, F.; Bearpark, M.; Heyd, J. J.; Brothers, E.; Kudin, K. N.; Staroverov, V. N.; Kobayashi, R.; Normand, J.; Raghavachari, K.; Rendell, A.; Burant, J. C.; Iyengar, S. S.; Tomasi, J.; Cossi, M.; Rega, N.; Millam, J. M.; Klene,

45 M.; Knox, J. E.; Cross, J. B.; Bakken, V.; Adamo, C.; Jaramillo, J.; Gomperts, R.; Stratmann, R. E.; Yazyev, O.; Austin, A. J.; Cammi, R.; Pomelli, C.; Ochterski, J. W.; Martin, R. L.; Morokuma, K.; Zakrzewski, V. G.; Voth, G. A.; Salvador, P.; Dannenberg, J. J.; Dapprich, S.; Daniels, A. D.; Farkas, O.; Foresman, J. B.; Ortiz, J. V.;

50 Cioslowski, J.; Fox, D. J. Gaussian 09, Revision A.02; Gaussian Inc.: Wallingford, CT, 2009.

55. T. Yanai, D. P. Tew and N. C. Handy, Chem. Phys. Lett., 2004, 393, 51-57.

56. D. Jacquemin, T. L. Bahers, C. Adamo and I. Ciofini, Phys. Chem. 55 Chem. Phys., 2012, 14, 5383-5388.

57. W. S. Rasband, http://rsb.info.nih.gov/ij/, 1997-2011. 